time consuming and its use is often limited by jejunal tube dysfunction. We aim to describe our outcomes using a "through the PEG" technique of jejunal extension placement.

Methods PEG-J placement in our unit is based upon the technique described by Berger et al in 1996. Briefly, a 28Fr PEG tube is inserted and an ultrathin endoscope $(4.5 \mathrm{~mm})$ is passed through the PEG tube into the stomach and deep into the small bowel. A guidewire is passed down the endoscope and the endoscope withdrawn leaving the guidewire in place. A 12Fr jejunal extension is passed over the guidewire and inserted fully until seated in the PEG tube. A retrospective review of all PEG-J procedures covering the period 2006-2010 was carried out. Patient demographics, procedure type and indication, sedation requirements and complication rates were recorded. Average tube patency was calculated for each patient (in days) and reason for tube replacement was recorded.

Results Over the study period, 121 procedures were carried out in 17 patients (mean age 59.6 years; $70.6 \%(n=12)$ males). Initial placement was successful in 120/121 (99.2\%) procedures with a procedure related complication rate of $1 / 121$ (0.8\%> bleeding). Indications for PEG-J placement were recurrent aspiration $(n=6)$, stroke $(n=2)$, neurodegenerative disorder $(n=2)$, gastroparesis $(n=2)$, post-operative $(n=1)$, oesophageal tear $(n=1)$, drainage $(n=1)$ and not documented $(n=2)$. 102/121 (84.3\%) procedures were for replacement of the jejunal extension tube alone. 73/102 (71.2\%) had no indication for tube replacement recorded. The most common causes of jejunal tube dysfunction were kinking $(n=12)$, occlusion $(n=8)$ tube breakage $(n=3)$, tube leakage $(n=3)$ and other $(n=3)$. The mean number of procedures per patient was 7.1 and the mean tube patency was 123.6 days. 90/121 (74.4\%) of procedures were performed without conscious sedation. 13/102 (12.7\%) jejunal replacements were performed under sedation vs $18 / 19$ (94.7\%) gastrostomy plus jejunal extension placements $(\mathrm{p}<0.001)$.

Conclusion "Through the PEG" placement of the jejunal extension is a safe and well tolerated procedure in what is often a difficult group of patients. Our technique confers high success rates of initial placement and low complication rates, with acceptable tube patency. Sedation is only occasionally required for those undergoing replacement of the jejunal extension.

Competing interests None declared.

\section{PM0-083 ARE WE MEETING THE NUTRITIONAL NEEDS OF OUR ACUTELY UNWELL SURGICAL INPATIENTS?}

doi:10.1136/gutjnl-2012-302514b.83

S Sarkar,* A Sandison. Department of General Surgery, East Sussex Hospitals, Eastbourne, UK

Introduction Patients, admitted to a surgical ward who have undergone a prolonged pre-admission illness are likely to have some element of malnutrition. This may be compounded by surgical procedures (and subsequent fasting) after admission and these patients can go into severe malnutrition quickly, often before the treating team realises it. The presence of malnutrition in a surgical patient has a direct bearing on the overall outcome during hospitalisation. A nutritionally deprived patient is unable to mount an adequate response against infection and the surgical outcome is hence likely to be sub-optimal. Complications such as intestinal anastomosis leakage, wound dehiscence and overwhelming sepsis are more common in patients with malnutrition. The British Society of Gastroenterology has recognised that such patients are common in UK hospitals and on average their length of stay is almost doubled. This had led to the development of comprehensive guidelines regarding enteral feeding in adult hospitalised patients. The aim of the present study was to compare current practice in a district general hospital to the BSG guidelines.

Methods A cross-sectional observational study of inpatients on general surgical wards over a 24 -h period was performed. Medical and nursing notes were reviewed and data recorded regarding nutritional assessment and management.

Results 72 patients were assessed over a 24-h period. Patients were divided into three groups, A elective surgery, B emergency surgery and $\mathrm{C}$ acute non-operative admission. Nutritional assessments were carried out on all patients, however BMI was not recorded in any. Pre admission nutritional intake was recorded in 61 (85\%) patients. Data from these 61 patients were analysed to determine period of starvation. The median number of days between starvation and commencement of nutritional support was three (range $0-7), 6$ $(0-11)$ and $5(0-12)$ respectively.

Conclusion This study has shown that many of the patients on a general surgical ward have a significant period of starvation prior to and during their admission. While a nutritional assessment was performed in all patients, BMI and thus assessment of malnourishment as advocated by the BSG guidelines was not recorded in any patient. The nutritional needs of elective surgical patients appears to be well met in our hospital (median time to feeding 3 days) when compared to the BSG guidelines (3-5 days). Emergency admissions, however, were not as well managed with many waiting up to 1 week before nutritional support was instigated. Adequate assessment of nutritional status is important in all surgical patients, this is equally important for both patients undergoing surgery and those who do not require any surgical intervention.

Competing interests None declared.

\section{PM0-084 THE EVOLUTION OF A VIRTUAL PEG ASSESSMENT TEAM: ACTIVITY AND COMPLICATIONS}

doi:10.1136/gutjnl-2012-302514b.84

S C Donnelly, ${ }^{*}$ R Sweis, V Sayer, L Bensaid, B Hayee. Department of Gastroenterology, Kings College Hospital, London, UK

Introduction Patients referred for percutaneous Endoscopic Gastrostomy (PEG) insertion often have multiple co-morbidities which do not improve with PEG feeding and lead to significant post-procedure complications. Pre-assessment of patients for PEG insertion improves morbidity and mortality, but is often time-consuming and labour-intensive. An electronic patient record (EPR) could facilitate assessment of patients and multi-disciplinary communication, while a detailed referral form could speed up the information gathering and assessment process. Starting in February 2011 in our Trust, patients referred for PEG were formally pre-assessed at the bedside by a Consultant Gastroenterologist and Nurse Endoscopist. In June 2011 a new electronic referral form was introduced. As a result of the improvements from February to May, a "virtual" assessment team including a Senior Dietician, Speech and Language Therapist, Elderly Care and Palliative Care physician was convened. The referral form was sent by secure email to the team and a virtual discussion took place with formal MDT meeting held when deemed prudent by consensus. Bedside assessment could then be restricted an assessment of fitness for the endoscopic procedure. Data were collected prospectively throughout the year and compared to practice in the preceding 12 months.

Methods Retrospective casenote analysis was conducted using the EPR and Endoscopy databases $\left(\right.$ Endosoft $^{\circledR}$ ) to include all PEG insertions performed. Prospective data collection were possible for all cases undergoing formal and virtual assessment.

Results In 2010, 96 PEG insertions were performed (median 6 per month), compared to 49 within the "formal" and "virtual" team periods (median 2 per month, $\mathrm{p}<0.005$ ). Patients did not differ significantly by age or gender. 30d mortality was $9 / 96$ (9.4\%) in 2010 and $2 / 49$ in 2011 (4.1\%; $p=0.33$ ). Rates of infection, aspiration pneumonia, buried bumper syndrome and other complications fell significantly $(p<0.001)$. The number of referrals not leading to insertion fell significantly between the formal and virtual assessment periods $(p<0.01)$ with no PEGs inserted in June and July. In 
addition, the time from referral to insertion increased significantly across these periods $(p<0.01)$. In the later part of 2011 an increase in referrals and appropriate insertions was observed-without a concomitant rise in complications.

Conclusion Introduction of a "virtual" team for PEG assessment reduced the number of procedures required (freeing time for other endoscopic procedures), and post-insertion complications. There was a non-significant trend for improved 30-day mortality. A "minimal input" approach to PEG assessment based on a detailed referral form is therefore feasible, safe and associated with significantly reduced rates of post-procedure morbidity.

Competing interests None declared.

\section{PM0-085 SAFETY AND SUBSEQUENT USE OF PROPHYLACTIC PERCUTANEOUS GASTROSTOMY PLACEMENT BY ENDOSCOPICALLY ASSISTED GASTROPEXY AND DIRECT PUNCTURE USING THE FRESENIUS ${ }^{\circledR}$ PEXACT KIT IN HEAD AND NECK CANCER PATIENTS}

doi:10.1136/gutjnl-2012-302514b.85

${ }^{1} \mathrm{~S} F$ Habib, ${ }^{1} \mathrm{O}$ Noorullah, ${ }^{1} \mathrm{~N}$ Stern, ${ }^{2} \mathrm{~S} N$ Rogers, ${ }^{1} \mathrm{~S}$ Ahmed. ${ }^{1}$ Department of Gastroenterology, University Hospital Aintree, Liverpool, UK; ${ }^{2}$ Maxillofacial Surgery, University Hospital Aintree, Liverpool, UK

Introduction Maintaining oral nutrition in Head and Neck cancer patients undergoing treatment can be challenging. Therefore, patients deemed at risk of malnutrition are referred for prophylactic gastrostomy. Due to risk of tumour implantation at gastrostomy site with conventional "pull though" technique, we changed our practice to direct puncture gastrostomy in 2004, using the Fresenius ( PEXACT kit. We have previously reported series of 319 patients. ${ }^{1}$ Methods All procedures performed between January 2010 and June 2011 were identified using the endoscopy reporting system. Information regarding readmissions, complications, mortality, dietetic assessment and use of gastrostomy tube was obtained from hospital patient records.

Results 91 gastrostomies were identified in 91 patients, 49 (54\%) had advanced T3/T4 cancers, 10 (11\%) with T2 disease. 69 (76\%) were males. Mean age $=55$ years (range $32-78$ ). Insertion was successful in all patients. All patients had prophylactic antibiotics prior to the procedure. There were no immediate procedure related complications (two immediate complications, one requiring a laparotomy, in cohort reported earlier, $n=319) .{ }^{1}$ There were no deaths within 7 days of procedure. Five patients died within 30 days (5.4\%). Four were due to disease progression, one patient had a cardiac arrest in the community 23 days after the procedure. There was 1 (1.09\%) unplanned admission 3 weeks after the procedure with bleeding from gastrostomy site requiring laparotomy. (14 unplanned admissions 30 days post procedure in earlier cohort, $n=319) .{ }^{1}$ There were no readmissions within 7 days. No cases of tumour implantation reported to date. Late displacement of gastrostomy tube ( $>30$ days after insertion) was common (6.5\%, same as in earlier cohort). ${ }^{1}$ To date we have managed to get information regarding use of gastrostomy tube in 58 out of 91 patients. Available data date so far has shown 46/58 (79.3\%) patients used their gastrostomy tube for 2 weeks or more. 12/58 (20.6\%) did not require use of gastrostomy tube.

Conclusion Endoscopically assisted gastropexy and direct puncture is a safe and reliable method of gastrostomy tube placement. Overall, our complication rate has fallen, with only one delayed major procedure related complication during this period. There have been no procedure related deaths or cases of tumour implantation.

Competing interests None declared.

\section{REFERENCE}

1. Ahmed S, Bowering K, Polavarapu N, et al. PEXACT®: analysis of 319 procedures performed at the digestive diseases unit, University Hospital Aintree. Gut 2010;59: A143. doi:10.1136/gut.2009.209049k

\section{PM0-086 HOW USEFUL IS FEEDING JEJUNOSTOMY IN UPPER GASTRO INTESTINAL CANCER SURGERY-A RETROSPECTIVE REVIEW}

doi:10.1136/gutjnl-2012-302514b.86

V Daya Shetty. ${ }^{*}$ Department of Upper GI Surgery, Salford Royal Hospitals NHS Foundation Trust, Bolton, UK

Introduction A retrospective review of outcome of 100 consectutive open feeding jejunostomies performed as part upper GI cancer surgery in our Centre in the last 3 years.

Methods 100 consecutive patients undergoing open insertion of Freka feeding jejunostomy as a part of Upper GI cancer surgery in the last 3 years are included. All feeding tubes were inserted approximately $30 \mathrm{cms}$ distal to the duodenojejunal flexure. The average procedure time for jejunostomy placement was $20 \mathrm{~min}$. The feeding jejunostomy was flushed with water on the night of surgery and a standardised feeding regime initiated used from fthe first postoperative day. The standard regime was water at $20 \mathrm{mls} / \mathrm{h}$ on day 1 followed by feed (Jevity/Osmolyte) at $30 \mathrm{mls} / \mathrm{h}$ on day 2 . The rate of feed was increased on daily increment of $10 \mathrm{mls} / \mathrm{h} /$ day to achieve target rate based on patient's nutritional requirements. All patients were discharged with feeding jejunostomy in situ. It was removed at first follow-up clinic appointment 2 weeks after discharge if patient was nutritionally stable.

Results A total of 100 patients (male: female=66:34) who had feeding jejunostomy tubes inserted are included. The indications were cardio-oesophagectomy (77\%); total gastrectomy (19\%); inoperable tumour at laparotomy $(3 \%)$ and in one patient prior to neoadjuvant chemotherapy. There were no intra operative procedure related complications. The median duration the feeding jejunostomy was in situ was 28 days (range 3-238 days). Postoperative feeding tube related complications were seen in $14 \%(n=14)$. These include tube fallout $(\mathrm{n}=5)$; minimal leak $(\mathrm{n}=2)$ and skin puncture site cellulitis $(n=7)$. Enteral feed related complications were seen in $15 \%$ $(n=15)$. These complications were minor and they included diarrhoea $(n=9)$, abdominal cramps and bloating $(n=4)$. Major complication was seen in only $6.8 \%(n=2)$ both due to feed (Jevity) forming a solid bezoar which caused small bowel obstruction. Laparotomy was necessary in one patient, with full recovery. The other the patient died following small bowel infarction. The availability of enteral route was particularly beneficial in 30 of our patients, to provide additional nutritional support for longer than anticipated, due to post operative difficulties including poor oral intake, anastamotic leak, and respiratory complications. In our series in only $5 \%(n=5)$ additional parenteral nutrition was necessary. This includes chyle leak- $(n=3)$ and dislodged feeding tube $(n=2)$.

Conclusion Feeding jejunostomy aids early establishment of enteral nutrition in patients undergoing upper GI cancer surgery. It is useful in providing continued nutritional support in patients who develop perioperative complications where oral route for nutrition is otherwise unavailable or inadequate, although jejunostomy tube placement and usage can also be a source of morbidity.

Competing interests None declared.

\section{PM0-087 LAPAROSCOPIC INSERTION OF FREKA FEEDING JEJUNOSTOMY AS A PART OF LAPAROSCOPIC THORACOSCOPIC CARDIO-OSOPHAGECTOMY-A REVIEW OF OUR OUTCOME}

doi:10.1136/gutjnl-2012-302514b.87

V Daya Shetty, ${ }^{*}$ K Akhtar. Department of Upper Gl Surgery, Salford Royal Hospitals NHS Foundation Trust, Salford, UK

Introduction A retrospective review of outcomes of laparoscopic insertion of feeding jejunostomy as a part of laparoscopic thoracoscopic cardio-oesophagectomy. 